\title{
Incumplimientos más habituales que comparten los estudios y estudios básicos de seguridad y salud en las obras de construcción de la Armada, respecto a las exigencias del Real Decreto 1627/1997 (1)
}

\section{More common defaults that share the studies and basic studies of safety and health in the construction works of the Navy, with respect to the requirements of Royal Decree 1627/1997 (1)}

$\underline{\text { M. A. Zapata Lobo }}^{(*),(* *)}$, A. Ros Serrano ${ }^{(* *)}$, P. Izquierdo Gracia ${ }^{(*)}$

\section{RESUMEN}

Esta investigación (2) surge del análisis de 248 informes de la Oficina de Supervisión de Proyectos la Dirección de Infraestructura de la Armada (3), correspondientes tanto a estudios como a estudios básicos de seguridad y salud, que han puesto de manifiesto la frecuente carencia de determinados aspectos documentales en los contenidos mínimos obligatorios que establece el Real Decreto 1627/1997.

Esta situación ha motivado un estudio minucioso sobre ello, analizando de forma detallada las incidencias más habituales para buscar su posible origen y comparando las que son comunes para ambos tipos de documentos.

Palabras clave: Estudio de seguridad; Construcción; Incumplimientos; Real Decreto 1627/1997.

\section{ABSTRACT}

This research (2) arises from the analysis of 248 reports from the Office of Project Supervision of the Navy Infrastructure Department (3), corresponding to both studies and basic safety and health studies, that have revealed the frequent lack of certain documentary aspects in the minimum mandatory contents established by Royal Decree 1627/1997.

This situation has led to a thorough study about it, analyzing in detail the most common incidents to find its possible origin and comparing those that are common to both types of documents.

Keywords: Safety study; Construction; Defaults; Royal Decree 1627/1997.

\author{
(*) Ministerio de Defensa (Armada Española). \\ (**) E.T.S. Edificación. Universidad Politécnica de Madrid (España). \\ Persona de contacto/Corresponding author: mzaplob@fn.mde.es (M. A. Zapata Lobo) \\ ORCID: http://orcid.org/oooo-0oo3-2208-4738 (M. A. Zapata Lobo); http://orcid.org/oooo-0oo3-1493-9126 (A. Ros \\ Serrano); http://orcid.org/oooo-0oo3-3425-2567 (P. Izquierdo Gracia)
}

\footnotetext{
Cómo citar este artículo/Citation: M. A. Zapata Lobo, A. Ros Serrano, P. Izquierdo Gracia (2018). Incumplimientos más habituales que comparten los estudios y estudios básicos de seguridad y salud en las obras de construcción de la Armada, respecto a las exigencias del Real Decreto 1627/1997 (1). Informes de la Construcción, 70(551): e263. https://doi.org/10.3989/ic.58255

Copyright: (c) 2018 CSIC. Este es un artículo de acceso abierto distribuido bajo los términos de la licencia de uso y distribución Creative Commons Reconocimiento 4.0 Internacional (CC BY 4.0).
} 


\section{INTRODUCCIÓN}

Transcurridos 20 años de la entrada en vigor del Real Decreto $1627 / 1997$ (RD 1627/97), se advierte que ciertos aspectos, que deberían haberse normalizado en los contenidos mínimos de los Estudios Básicos de Seguridad y Salud (EBSS) y los Estudios de Seguridad y Salud (ESS), siguen sin ser contemplados, son desarrollados parcialmente o son inadecuadamente tratados.

De los resultados de este análisis se desprende que, la exigencia de incluir el EBSS o ESS como un documento más el proyecto, establecida en el Artículo 4 del citado RD, se trata con frecuencia como un mero trámite administrativo en lugar de prestar la debida atención al obligado cumplimiento de un adecuado alcance y desarrollo en los contenidos mínimos de estos documentos.

Es esencial comprender que estos documentos requieren un especial tratamiento y un cuidado desarrollo. Deben ser redactados por "técnicos competentes" (4) (5) conforme a los Artículos 5 y 6 del RD 1627/97, aunque el legislador deje en la ambigüedad esta figura al no detallar las titulaciones habilitantes para ello en ninguna normativa.

Estos profesionales deben disponer de los conocimientos técnicos precisos para comprender el alcance de la intervención, el proceso constructivo, los materiales empleados, los medios y los procedimientos necesarios para su puesta en obra, así como de la organización, planificación y programación de la actuación.

La ausencia de estos conocimientos puede ser una de las causas de los resultados obtenidos en este análisis, pero más allá de la discusión de competencias y atribuciones profesionales, este trabajo busca poner de manifiesto las deficiencias más habituales en los contenidos de los EBSS y ESS, en base a las exigencias mínimas del RD 1627/97 y su adecuado desarrollo.

Sucede también, que a la hora de elaborar dichos documentos, se obvia con frecuencia el carácter de mínimos que el propio título del RD 1627/1997 establece y que dimana del Artículo 2.2. de la Ley 31/1995 (6), donde se establece que "Las disposiciones de carácter laboral contenidas en esta Ley y en sus normas reglamentarias tendrán en todo caso el carácter de Derecho necesario mínimo indisponible, pudiendo ser mejoradas y desarrolladas en los convenios colectivos.", eludiendo la inclusión de la información realmente necesaria, bajo la excusa de que no se precisa al no ser un requisito expreso de los mínimos legalmente exigibles.

Igualmente, es un defecto generalizado el mal uso de las herramientas comerciales de carácter informático para elaborar estos documentos, especialmente en los ESS. Pese a que estas herramientas generan documentos que contemplan la casi totalidad de las exigencias normativas, una vez cumplimentados con los datos de la actuación, no quedan adaptados a la particularidad de la intervención, resultando documentos genéricos que carecen de una aplicación real y eficaz para la actividad preventiva de la obra.

Del mismo modo, los modelos que existen para elaborar los EBSS, carecen de gran parte de los contenidos exigibles y necesarios para que el documento resultante contemple todo lo preciso desde el punto de vista de la actuación, así como todo lo requerido desde el punto de vista normativo. Como sucede con los ESS, no se suele concretar el documento final a la particularidad de la obra, siendo igualmente genéricos y sin la posibilidad de una aplicación real y eficaz a cada intervención.

\section{OBJETIVOS DE LA INVESTIGACIÓN}

\subsection{Objetivo general}

Examinar los contenidos de los informes de supervisión de EBSS y ESS, comprobando las carencias más habituales en cada tipología de documento conforme a las exigencias mínimas que establece en RD 1627/97, y con ello realizar un estudio comparativo de los apartados comunes entre ambas tipologías EBSS y ESS, analizando las posibles causas que los motivan.

\subsection{Objetivos específicos}

2.2.1. Identificar los contenidos mínimos exigibles en los EBSS, en base a lo establecido en el RD 1627/97

2.2.2. Identificar los contenidos mínimos exigibles en los ESS, comunes con los EBSS, en base a lo establecido en el RD 1627/97

2.2.3. Cuantificar los incumplimientos de los EBSS en referencia a los contenidos mínimos identificados en el punto 2.2.1

2.2.4. Cuantificar los incumplimientos de los ESS en referencia a los contenidos mínimos identificados en el punto 2.2.2

2.2.5. Realizar un estudio comparativo de los contenidos comunes, identificados en los puntos 2.2.1. y 2.2.2., respecto de las carencias más habituales determinadas en los puntos 2.2.3. y 2.2.4

2.2.6. Concluir las posibles causas que originan dichos incumplimientos en base al contenido de los informes de supervisión analizados

\section{METODOLOGÍA EMPLEADA}

Para esta investigación se utilizan dos métodos de trabajo:

\subsection{Análisis cualitativo}

Consiste en identificar los contenidos mínimos comunes para ambos tipos de documentos EBSS y ESS, en base a los requisitos de los Artículos 6 y 5 del RD 1627/97 respectivamente y apoyado con alguna exigencia común más del mismo RD.

Se genera la Tabla 1, cuya cabecera se ordena con los siguientes conceptos: Número de Incidencia [N.I.] / Articulado que supone la exigencia de cumplimiento para los EBSS / Articulado que supone la exigencia de cumplimiento para los ESS / Resumen del alcance.

\subsection{Análisis cuantitativo}

Consiste en ponderar los incumplimientos de cada exigencia normativa. Se analizan por separado cada una de dichas exigencias, tanto para los EBSS como para los ESS, y paralelamente se realiza un estudio comparativo entre ambos tipos de 
Tabla 1. Exigencias normativas mínimas para los EBSS y ESS.

\begin{tabular}{|c|c|c|c|}
\hline \multicolumn{4}{|c|}{ Referencias al articulado del RD 1627/97 } \\
\hline N.I. & EBSS & ESS & Resumen del alcance \\
\hline 1 & Artículo 4.1. & Artículo 4.1. & Justificación de los 4 supuestos con objetividad. \\
\hline 2 & Artículo 6.2. & Artículo 5.2.b. & Normativa de seguridad y salud aplicable a la obra. \\
\hline 3 & Artículo 6.2. & Artículo 5.2.a. & Riesgos laborales que puedan ser evitados. \\
\hline 4 & Artículo 6.2. & Artículo 5.2.a. & Riesgos laborales que no puedan eliminarse. \\
\hline 5 & Artículo 6.2. & Artículo $5 \cdot 5$ & Riesgos especial peligrosidad - Actividades del anexo II. \\
\hline 6 & Artículo 6.3. & Artículo 5.6. & Previsiones e informaciones para los previsibles trabajos posteriores. \\
\hline 7 & Artículo 8.1.a. & Artículo 8.1.a. & $\begin{array}{l}\text { Decisiones para planificar los trabajos o sus fases simultáneas o } \\
\text { sucesivas. }\end{array}$ \\
\hline 8 & Artículo 8.1.b. & Artículo 8.1.b. & $\begin{array}{l}\text { Estimación de la duración para la ejecución de los distintos trabajos } \\
\text { o sus fases. }\end{array}$ \\
\hline 9 & Artículo 15. Anexo IV-Parte A-Punto 14 & Artículo 5.2.a. & Instalaciones sanitarias y centros asistenciales. \\
\hline 10 & Artículo 15. Anexo IV-Parte A-Puntos 15 y 16 & Artículo 5.2.a. & Instalaciones de higiene y bienestar. \\
\hline 11 & Artículo 15. Anexo IV-Partes A, B y C. & Artículo 5.2.c. & Documentación gráfica. \\
\hline
\end{tabular}

documentos para cada incumplimiento, interpretando así las posibles causas de los mismos en cada caso.

Los datos comparativos de la Tabla 2 y Gráfico 1, han sido ordenados de mayor a menor número de incumplimientos en conjunto, EBSS y ESS.

Se identifica como «Número de Incidencia Cero» [N.I.o] a los documentos aceptados y que han contemplado los requisitos del análisis cualitativo. Al no tratarse de un incumplimiento como tal, sus porcentajes no guardan orden con el resto de datos y figura siempre en primer lugar.

\section{TAMAÑO DE LA MUESTRA Y FUENTE.}

Para esta investigación se han analizado 248 informes, desde el año 2001 al año 2016 ambos inclusive, que corresponden a 65 EBSS y 183 ESS.
Los documentos se han obtenido del archivo de la Oficina de Supervisión de Proyectos de la Dirección de Infraestructura de la Armada.

Desde antes del año 200o, la Armada viene externalizando la elaboración de proyectos con distintas empresas. Consecuentemente, la redacción de EBSS y ESS se realiza por personal ajeno a la Armada. Solo de forma excepcional, algunos proyectos son elaborados por personal propio.

La casi totalidad de las actuaciones corresponden a obras de primer establecimiento, reformas y adecuaciones, siendo una excepción las actuaciones de rehabilitación o demolición.

\section{RESULTADOS}

Del análisis de los informes, se desprenden los siguientes resultados.

Tabla 2. Comparativa de cantidades y porcentajes de incumplimientos

\begin{tabular}{|c|c|c|c|c|c|c|c|c|}
\hline \multicolumn{9}{|c|}{ COMPARATIVA ENTRE ESTUDIOS BÁSICOS Y ESTUDIOS DE SEGURIDAD } \\
\hline \multirow[b]{2}{*}{ N.I. } & \multirow[b]{2}{*}{ Concepto } & \multicolumn{3}{|c|}{ EBSS } & \multicolumn{3}{|c|}{ ESS } & \multirow{2}{*}{$\begin{array}{l}\text { Cantidad de } \\
\text { incumplim. } \\
\text { en conjunto }\end{array}$} \\
\hline & & $\begin{array}{c}\text { Cantidad } \\
\text { incumplim. }\end{array}$ & \begin{tabular}{|c|}
$\%$ \\
incumplim.
\end{tabular} & & \begin{tabular}{|c|} 
Cantidad \\
incumplim.
\end{tabular} & $\begin{array}{c}\% \\
\text { incumplim. }\end{array}$ & & \\
\hline O & ACEPTADOS & 3 & $4,62 \%$ & (i) & 15 & $8,20 \%$ & (iii) & 18 \\
\hline 8 & PROGRAMA DE TRABAJOS & 62 & $100,00 \%$ & \multirow{11}{*}{ (ii) } & 152 & $90,48 \%$ & \multirow{11}{*}{ (iv) } & 214 \\
\hline 1 & JUSTIFICACIÓN & 49 & $79,03 \%$ & & 112 & $66,67 \%$ & & 161 \\
\hline 3 & RIESGOS EVITABLES & 31 & $50,00 \%$ & & 98 & $58,33 \%$ & & 129 \\
\hline 11 & PLANOS & 42 & $67,74 \%$ & & 82 & $48,81 \%$ & & 124 \\
\hline 10 & HIGIENE Y BIENESTAR & 25 & $40,32 \%$ & & 95 & $56,55 \%$ & & 120 \\
\hline 4 & RIESGOS NO ELIMINABLES & 32 & $51,61 \%$ & & 83 & $49,40 \%$ & & 115 \\
\hline 9 & INSTALACIONES SANITARIAS & 35 & $56,45 \%$ & & 55 & $32,74 \%$ & & 90 \\
\hline 5 & RIESGOS ESPECIALES & 23 & $37,10 \%$ & & 63 & $37,50 \%$ & & 86 \\
\hline 6 & TRABAJOS POSTERIORES & 19 & $30,65 \%$ & & 59 & $35,12 \%$ & & 78 \\
\hline 7 & ORGANIZACIÓN-PLANIFICACIÓN & 18 & $29,03 \%$ & & 26 & $15,48 \%$ & & 44 \\
\hline 2 & NORMATIVA & 21 & $33,87 \%$ & & 22 & $13,10 \%$ & & 43 \\
\hline
\end{tabular}

(i) Referido al total de EBSS 65

(ii) Referido a los EBSS rechazados 62

(iii) Referido al total de ESS 183

(iv) Referido a los ESS rechazados 168 


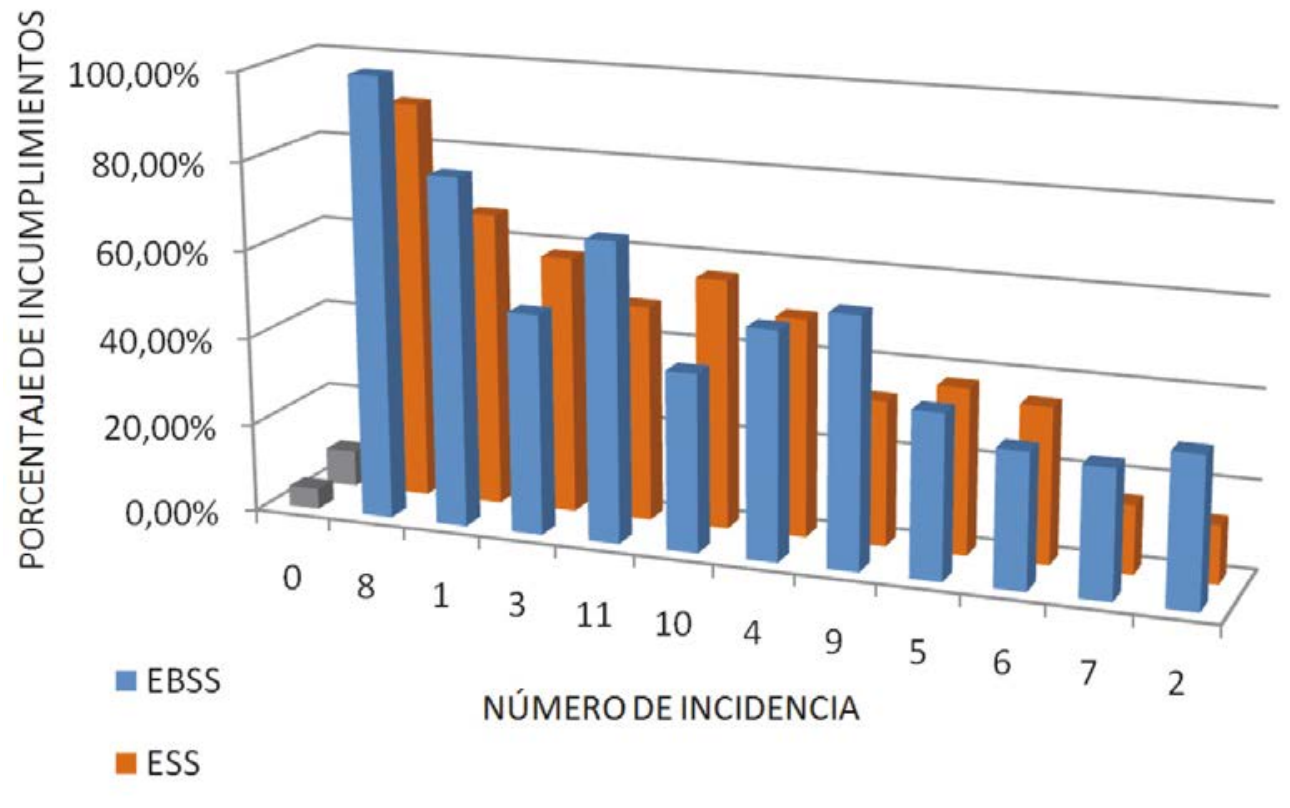

DOCUMENTOSACEPTADOS

Gráfico 1. Comparativo de porcentajes de incumplimientos.

\subsection{Análisis de los informes de supervisión de los estudios y estudios básicos de seguridad y salud}

De un total de 248 documentos:

- 18 Fueron aceptados en primera instancia por contemplar todos los requisitos definidos.

- Los 230 documentos restantes presentaron las siguientes carencias, ordenadas de mayor a menor cantidad de incumplimientos en conjunto de los EBSS y ESS:

- 214 No incorporan un programa de trabajos específico para la actuación.

- 161 No realizan o realizan de forma inadecuada, el análisis de los 4 supuestos del Artículo 4.1. del RD 1627/97 para justificar la elaboración de un EBSS o un ESS.

Como consecuencia de ello:

- 133 Precisaron modificar sus cálculos, alterando la previsión original de número de trabajadores, de los cuales, 14 EBSS debieron elaborarse nuevamente como ESS.

- 28 Precisaron modificar sus cálculos sin necesidad de alterar las previsiones originales.

- 129 No incorporan documentación gráfica o lo hacen de forma inadecuada o incompleta.

- 124 No facilitan la información sobre los servicios asistenciales y/o no definen las instalaciones sanitarias precisas para la intervención, o lo hacen de forma inadecuada o incompleta.

- 120 Identifican los riesgos laborales que no puedan eliminarse, pero no valoran la eficacia de las medidas preventivas que se proponen.

- 115 No identifican los riesgos laborales que puedan ser evitados, o lo hacen de forma inadecuada o incompleta.

- 90 No definen las instalaciones de higiene y bienestar precisas, o lo hacen de forma inadecuada o incompleta.

- 86 No identifican la existencia de riesgos especiales, o lo hacen de forma inadecuada o incompleta.

- 78 No incluyen la normativa, o lo hacen de forma inadecuada o incompleta.
- 44 No contemplan las previsiones e informaciones para los previsibles trabajos posteriores, o lo hacen de forma inadecuada o incompleta.

- 43 No realizan una adecuada organización y planificación de la actividad preventiva, o lo hacen de forma inadecuada o incompleta.

Con los resultados obtenidos se realiza un análisis individualizado a la vez que comparativo.

Para ello se elabora la Tabla 2 y el Gráfico 1, con los siguientes contenidos:

Tabla 2 Refleja los datos de EBSS y ESS comparativos, indicando: Número de Incidencia [N.I.] / Concepto resumido / Cantidad de incumplimientos de cada incidencia en los EBSS / Porcentaje de dichos incumplimientos en los EBSS / Cantidad de incumplimientos de cada incidencia en los ESS / Porcentajes de dichos incumplimientos en los ESS / Cantidad de incumplimientos en conjunto.

Gráfico 1 Diagrama de barras comparativo de los datos de la (Tabla 2) correspondientes al Número de Incidencia y al porcentaje de incumplimientos de EBSS y ESS.

\subsection{Discusión de los resultados}

El orden de exposición de este apartado mantiene el criterio seguido en la Tabla 2, siendo considerados de mayor a menor cantidad de incumplimientos en conjunto.

Ocasionalmente, ciertos incumplimientos surgen por una casuística variada que no se cuantifica. Esto sucede porque los informes reflejan el incumplimiento normativo, pero no siempre el motivo expreso que lo genera, de modo que al no poderse cualificar con el rigor suficiente como para ofrecer un valor fiable para el análisis, se omite del mismo. 
Antes de comenzar con el análisis pormenorizado de las incidencias, conviene aclarar que el Artículo 15 del RD 1627/97, que se cita en las incidencias 9, 10 y 11 de los EBSS, habla de que "los contratistas y subcontratistas deberán garantizar que los trabajadores reciban una información adecuada de todas las medidas que hayan de adoptarse en lo que se refiere a su seguridad y su salud en la obra", lo cual debe hacerse en el PSS, documento que se desarrolla en base a las previsiones que se establecen en el EBSS o ESS.

Esto supone que dichos EBSS o ESS deben contemplar y especificar las previsiones de dichas medidas, las cuales se definen de forma particularizada en las distintas partes A, B y C del anexo IV. Por este motivo, hay una vinculación clara y directa entre la aplicación de estos apartados en desarrollo del citado artículo 15, para establecer unos criterios, en este caso en los EBSS por parte del proyectista, para que el contratista pueda desarrollaros correctamente.

\section{[N.I.o] Aceptados:}

El índice de documentos aceptados en general es muy bajo, 4,62\% de EBSS y 8,20\% de ESS. La diferencia estriba en que los ESS corresponden generalmente a obras de mayor entidad o complejidad, lo que unido a la mayor exigencia de contenidos en el documento, hace que su dedicación y detalle a la hora de ser redactados sea mayor, y como consecuencia sean documentos más completos y elaborados.

El índice de incumplimientos se detalla pormenorizadamente en los siguientes apartados.

\section{[N.I.8] Programa de trabajos:}

Este aspecto presenta el mayor índice de incumplimiento, el 100\% en EBSS y el 90,48\% en ESS, interpretándose, que al no ser una exigencia expresa de los Artículos 6 y 5, ninguno de los documentos lo requieren, eludiendo que este punto debe desarrollarse en aplicación del Artículo 8 de RD 1627/97, en especial su apartado b).

Los proyectos de obras que se elaboran para la Administración Pública, requieren la incorporación de un programa de desarrollo de trabajos, en cumplimiento del Artículo 233, punto 1, apartado e) de la Ley 9/2017 (7).

En los proyectos que requieren la elaboración de EBSS, se suele excluir este programa de trabajos de los preceptivos documentos del proyecto en aplicación del Artículo 233, punto 2 de la citada Ley 9/2017, complicando esta labor en los EBSS y aumentando así su índice de incumplimientos respecto a los que desarrollan un ESS.

Es habitual interpretar que el nivel de detalle en contenidos de los EBSS y ESS depende de la envergadura de la obra, error que sucede al no comprender que el riesgo es propio de cada actividad, independientemente de la obra en que se realice.

Por otra parte, cuando se cuantifica en el programa de trabajos del proyecto el capítulo de seguridad y salud en el conjunto de la obra, se hace de forma permanente y uniforme durante toda la actuación, lo que es irreal.

Al inicio de la obra, la inversión en prevención es notablemente mayor, ya que es el momento en que se dispone de las instalaciones de higiene y bienestar, se establece el perímetro y acceso de la obra, se facilitan la mayor parte de los equipos de protección individual, se disponen generalmente gran parte de los medios auxiliares que se utilizan con finalidad preventiva y se acometen las actuaciones de primera intervención, definidas como "riesgos laborales que puedan ser evitados".

No aplicar estos criterios supone realizar certificaciones que no se corresponden con la realidad en el capítulo de seguridad y salud de la obra.

El programa de trabajos de seguridad y salud debe ser complementario al programa de trabajos del proyecto, debiendo detallar la actividad preventiva con rigor y en paralelo con el resto de actividades de la obra. Se debe establecer en qué momento se prevé el montaje y desmontaje de las protecciones colectivas en cada fase, cuándo se delimitará el recinto de la actuación, se establecerán las zonas de trasiego, acopios, accesos, ubicación de contenedores, instalaciones sanitarias y comunes, así como estimar el número de trabajadores en cada una de sus fases o número pico de trabajadores en hitos específicos, adaptándolo en función de la actividad y evolución de la obra.

Este documento es sin duda una herramienta de extraordinaria eficacia, ya que nos permite determinar y comprobar, en un solo vistazo, la intensidad en la que hay que actuar en cada etapa de la obra y las necesidades preventivas en cada momento de la actuación (9) (10).

Aunque aparentemente este documento es más propio del Plan de Seguridad y Salud (PSS) que de un ESS o EBSS, la función del PSS es detallarlo y particularizarlo en función de los recursos del contratista, pero en base a las previsiones recogidas en el ESS o EBSS, cuando se precise.

Por ello, debe incorporarse en todas las intervenciones donde exista una simultaneidad de oficios que precise una especial coordinación entre sus espacios y actuaciones, cuando exista una interferencia con la actividad de la dependencia o cuando en la actuación se acometan trabajos con especial peligrosidad, como el manejo de grandes prefabricados o el uso de productos o residuos tóxicos o contaminantes.

\section{[N.I.1] Justificación:}

El incumplimiento de este apartado es muy elevado, del $79,03 \%$ en los EBSS y del 66,67\% en los ESS. Solo en el $33,33 \%$ de los ESS, el redactor utiliza los datos del proyecto para realizar estos cálculos. En el resto de casos, se consignan los valores de repercusión de mano de obra, precio medio de mano de obra directa, o incremento de punta de trabajo, de forma arbitraria, sin rigor ni criterio alguno.

Esto se constata por el alto índice de ocasiones en que resultaron modificadas las previsiones del número de trabajadores consignados inicialmente, 87 de los 112 ESS en los que se realizó el cálculo inadecuadamente.

En los EBSS, cuando el proyecto no refleja los datos precisos para este análisis, también se consignan de forma arbitraria y sin rigor en el 79,03\% de los documentos, en lugar de acudir a datos estadísticos de fuentes de solvencia, considerando el convenio del sector. 
Las previsiones iniciales del número de trabajadores se vieron alteradas en el 93,88\%, 46 de los 49 EBSS en los que se realizó el cálculo inadecuadamente, debiendo ser redactados nuevamente como ESS el 30,43\%, 14 de esos 46 EBSS.

En estos casos, el redactor consigna deliberadamente valores con el fin de no incurrir en ninguno de los supuestos que le supondrían elaborar un ESS. Esto se deduce por la enorme disparidad entre el dato consignado inicialmente y el dato obtenido posteriormente mediante un cálculo justificado, lo que se constata al observar que esos datos iniciales tan discrepantes dan como resultado un cumplimiento en el límite de las exigencias establecidas.

La estimación del número máximo de trabajadores es fácilmente obtenible en el caso de los ESS, ya que el programa de trabajos nos permite observar y cuantificar el incremento en punta de trabajo.

En los EBSS es más complejo, por la explicada posible ausencia del programa de trabajos, aunque menos relevante, dado que el carácter menor de estas intervenciones hace que el número medio de trabajadores obtenido en los EBSS sea notablemente inferior al que se obtiene en los ESS. Consecuentemente las repercusiones de punta de trabajo no alteran significativamente el resultado del número máximo de trabajadores con estimaciones estadísticas.

\section{[N.I.3] Riesgos evitables:}

Estos riesgos corresponden a las denominadas medidas de primera intervención, que tras su implantación al inicio de la obra, hacen que un determinado riesgo desaparezca. Esto supone su eliminación de la lista de riesgos no eliminables, cosa que no siempre sucede.

En los EBSS tiene una incidencia algo menor, el 50,00\%, frente al $58,33 \%$ de los ESS, lo que se justifica porque en los EBSS el análisis de los riesgos que no pueden eliminarse suele ser notablemente más escaso que en los ESS.

Los EBSS suelen formar parte de intervenciones que se realizan sobre edificios existentes, donde la posibilidad de afección a suministros y sus instalaciones o de interferencia con otro tipo de actividades, es más frecuente.

Estas situaciones suelen resolverse con dichas actuaciones de primera intervención, lo que supone un mayor énfasis en el análisis de este tipo de riesgos evitables, minorando así sus incumplimientos.

Ocasionalmente en los ESS, estos riesgos evitables son incluidos como riesgos no eliminables, precisamente por esta menor casuística, que hace que se considere apropiado agruparlos en un único análisis, lo que desde el punto de vista preventivo es un error puesto que su tratamiento es diferente.

También se utiliza la justificación de que este tipo de riesgos nunca existen para excluirlos de su identificación y análisis, circunstancia altamente improbable, ya que determinadas situaciones, como la interferencia de tráfico en circulaciones o accesos, o la existencia de instalaciones en servicio suelen ser inevitables en cualquier tipo de obra.

\section{[N.I.11] Planos:}

En esta incidencia hay una notable diferencia entre los EBSS, con el $67,74 \%$, y los ESS con un 48,81\%, ya que la exigencia de la inclusión de planos en el documento de seguridad y salud es un requisito expreso del Artículo 5 para los ESS.

Se interpreta erróneamente que, al no figurar en las exigencias del Artículo 6 del RD 1627/97, este requisito suele omitirse en los EBSS, sin considerar la necesidad de dar cumplimiento al desarrollo del Anexo IV, del RD 1627/97, en base al Artículo 15 del mismo RD, para definir correctamente la actividad preventiva, considerando así las disposiciones mínimas de seguridad y de salud que deberán aplicarse en las obras, las relativas a los puestos de trabajo en el interior de los locales y las relativas a puestos de trabajo en el exterior de los locales, según contemplan respectivamente las Partes A, B y C del citado Anexo IV.

En el caso de los ESS, los incumplimientos suceden por la carencia de planos de protecciones colectivas en cada una de las fases de la obra, o por omitir información relevante en el plano general de ordenación de la obra.

También se suele omitir la localización e identificación de las zonas en las que se llevan a cabo trabajos que implican riesgos especiales para la seguridad y la salud de los trabajadores, o la disposición y recorrido de suministros existentes en el área de la obra y que pueden verse afectados o pueden afectar a la actividad.

\section{[N.I.10] Higiene y bienestar:}

Este incumplimiento sucede por dos circunstancias distintas, de ahí que no tenga una vinculación directa en ambos casos con el [N.I.1-Justificación], que le afectaría directamente por el incorrecto cálculo del número máximo de trabajadores.

En el caso de los EBSS, el incumplimiento del 40,32\% radica en la falta de comprobación de la idoneidad de las instalaciones existentes en los inmuebles donde se va a actuar. Este hecho viene marcado por el mismo motivo que en el [N.I.9Instalaciones Sanitarias] al no ser una exigencia expresa del Artículo 6, sino del Artículo 15, en desarrollo de los puntos 15 y 16 del Anexo IV, Parte A sobre servicios higiénicos y locales de descanso, respectivamente.

El marcado carácter menor de estas intervenciones, que corresponden generalmente a obras de adecuación, reforma o reparación en inmuebles en funcionamiento, supone que se pueda disponer frecuentemente cerca de la actuación de aseos, vestuarios o espacios para descanso.

En estos casos, ese 40,32\% de los EBSS, se limitan a indicar que serán utilizadas dichas dependencias, lo cual es lícito, siempre y cuando sea compatible con la actividad de la dependencia y se tenga autorización del Centro, pero el incumplimiento viene por la ausencia de la comprobación de si las superficies y dotaciones de dichas instalaciones son aptas para el número máximo de trabajadores.

En estos casos sucede que, las superficies y/o dotaciones que se asignan a dichas instalaciones no se corresponden con el número máximo de trabajadores calculados, asignando mó- 
dulos estándar tomando erróneamente el número medio de trabajadores, o siendo asignados en función de un número de trabajadores calculados de forma inadecuada.

\section{[N.I.4] Riesgos no eliminables:}

En esta ocasión el grado de incumplimiento es muy similar, el 51,61\% en EBSS frente al 49,40\% en ESS, porque el motivo es el mismo. En ambos casos, los riesgos se identifican, pero no se valora la eficacia de las medidas preventivas que se proponen para ello.

Además, en un número que no se ha podido cuantificar, los informes mencionan que el redactor confunde la valoración de la eficacia de las medidas preventivas con la evaluación inicial de riesgos, que es motivo del PSS.

En ocasiones se incluyen fichas que, tras definir una actividad e identificar un riesgo concreto, valoran conjuntamente la eficacia de todas las medidas preventivas que se proponen, ya sean una o varias protecciones individuales o colectivas.

Este procedimiento no permite determinar la eficacia real de cada una de ellas, ni comprobar el principio preventivo de prevalencia de las medidas colectivas frente a las individuales, conforme a lo establecido en la Ley 31/1995 de PRL es su artículo 15, punto1, apartado $\mathrm{h}$.

\section{[N.I.9] Instalaciones sanitarias:}

Este incumplimiento es más habitual en los EBSS, con el $56,45 \%$ de los casos, que en los ESS con el 32,74\%. Sucede algo análogo al [N.I.11-Planos]. Al no tratarse de un requisito expreso como exigencia del Artículo 6, sino del Artículo 15, en desarrollo del punto 14 del Anexo IV, Parte A, sobre primeros auxilios, es obviado con gran frecuencia en los EBSS.

Las características y dotaciones de estas instalaciones, en la mayoría de los EBSS, se reducen a la existencia de botiquines portátiles de obra y a la identificación de los centros asistenciales más cercanos, lo que no se hace o se hace de modo inadecuado.

En los ESS, ese 32,74\% de incumplimientos obedece a que las dotaciones no se han definido en función del número máximo de trabajadores o del plazo de ejecución, aunque correspondan generalmente a botiquines portátiles y su reposición. También es consecuencia de no identificar los centros asistenciales más cercanos.

Habitualmente se argumenta que este aspecto de los centros asistenciales es materia del PSS. En este sentido debe advertirse que en el EBSS o ESS, es suficiente con que se identifiquen dichos centros, con su nombre, dirección, teléfono y tipo de asistencia, dejando constancia únicamente de su existencia y facilidad de acceso.

El PSS deberá detallar, completar y ampliar dicha información, desarrollando un plano con su localización, itinerario y ruta más rápida, distancia, tiempo estimado de llegada, alcance de las asistencias posibles, dirección exacta, teléfonos de contacto y urgencias, así como todos los demás detalles precisos para su rápida y fácil localización e identificación.

\section{[N.I.5] Riesgos especiales:}

Este incumplimiento, que sucede en el 37,50\% de los ESS, tiene un cierto paralelismo con los motivos del [N.I.2-Riesgos evitables].

Se consignan estos riesgos junto con los riesgos no eliminables. Esto elude la importancia de tratar de forma clara y diferenciada este tipo de riesgos, los cuales están indicando al contratista que el PSS debe ser especialmente cuidadoso con ellos, y que requieren de un tratamiento más minucioso y detallado que el resto de riesgos.

En el caso de los EBSS, ese 37,10\% de incumplimientos sucede por circunstancias análogas. Se agrupa con el resto de riesgos sin hacer esa distinción, motivo por el que el índice de incumplimiento es tan similar, del $37,10 \%$ en los EBSS y del $37,50 \%$ en los ESS.

También se observa en ambos tipos de documentos, que cuando estos riesgos no se presentan, no se indica de forma expresa, dejando la duda de si realmente no existen, o se ha omitido su inclusión.

\section{[N.I.6] Trabajos posteriores:}

En esta ocasión, el índice de incumplimiento es superior en los ESS, en el 35,12\% de los casos, frente al 30,65\% de las ocasiones en los EBSS, lo que puede parecer incongruente.

Los proyectos que desarrollan un ESS suelen precisar de la instalación de protecciones que pueden ser aprovechadas para el posterior mantenimiento del edificio si se dejan instaladas. En cambio, suelen ser desmontadas por lo general si valorar esta opción. Este hecho, unido a las ocasiones en que no se tiene en consideración su previsión expresa para este fin, hace que el índice de incumplimientos aumente.

En el caso de los EBSS, dado que estas actuaciones menores no suponen habitualmente la instalación de estas medidas que posiblemente pudiesen ser utilizadas para este fin, incluso pueden ya existir al actuarse en edificios que están en servicio, se definen y detallan con más rigor.

En ambos tipos de documentos tampoco se diferencia entre previsiones e informaciones, reduciéndose, en la mayoría de los incumplimientos, a consignar instrucciones genéricas como información, sin la correspondiente propuesta técnica de las previsiones precisas.

Igualmente se observa la carencia de considerar, dentro de las propuestas e informaciones, la facilidad, medios, instrucciones o elementos necesarios para poder acceder a los lugares que precisan este tipo de actuaciones.

\section{[N.I.7] Organización-Planificación:}

Dado que todos los proyectos precisan de un programa de trabajos, con la excepción ya citada en aplicación del Artículo 233, punto 2 de la Ley 9/2017, la organización y planificación suele ser incluida con gran frecuencia y precisión.

La magnitud de la intervención marca la diferencia. En las obras de mayor entidad y que suelen precisar ESS, concurren un mayor número de actividades y oficios, por lo que se presta una mayor atención al aspecto organizativo. 
En las intervenciones de menor magnitud que precisan de un EBSS y que pueden carecer de un programa de trabajos, se descuida algo más este desarrollo, de ahí que tenga un mayor índice de incumplimientos en los EBSS con el 29,03\%, frente al 15,48\% de los ESS.

\section{[N.I.2] Normativa:}

El motivo de este incumplimiento es idéntico en ambos casos, aunque en los EBSS suceda en el 33,87\% de las ocasiones y el los ESS solo en el 13,10\%. Esta diferencia se justifica debido a la obligatoriedad de elaborar un pliego de condiciones en el ESS que ya contempla necesariamente la normativa de aplicación, y pese a que en el EBSS también es una exigencia, suele omitirse con cierta frecuencia.

El incumplimiento común sucede por la omisión de la normativa específica, tanto del ámbito de la Administración General del Estado, como del Ministerio de Defensa en esta materia, generalmente en reglamentos de ámbito restringido, aunque de público acceso, pero que afectan de forma directa al correcto desarrollo de la actividad preventiva de la obra en Instalaciones Militares.

La más habitual, a la que se refiere en el 100\% de los casos de este incumplimiento, es la Instrucción 55/2011 del SEDEF (10) que, entre otros aspectos, regula la relación durante el desarrollo de la obra, entre la dependencia y el centro de trabajo que se apertura, actuando a modo de coordinación de actividades empresariales entre la actividad propia de la obra y la actividad de la dependencia para las actuaciones previas al comienzo de las obras.

Igualmente se incide en el carácter generalista de los listados que se incluyen, haciendo referencia a todo tipo de actividades, sin particularizar en cada intervención, debiendo incluir solo las que son de aplicación en cada caso.

\section{CONCLUSIONES}

1. Los EBSS y ESS deben ser elaborados por técnicos competentes, capaces de comprender la dimensión real de la aplicación de la actividad preventiva de la obra. El legislador debería definir con concreción y sin lugar a dudas, las titulaciones habilitantes para redactar los EBSS y ESS en cada tipo de intervención.

2. Tanto las herramientas comerciales de carácter informático como los formularios tipo para la elaboración de EBSS o ESS, pierden su eficacia si no se particularizan para cada actuación específica. No es admisible la entrega de documentos genéricos.

3. Los EBSS y los ESS no son un trámite administrativo para completar los documentos que componen un proyecto. Estos documentos requieren un desarrollo profesional, minucioso y particularizado para cada intervención.

4. El programa de trabajos del proyecto debe reflejar con rigor las aplicaciones económicas del capítulo de seguridad y salud durante la ejecución de la obra, en el momento en que realmente se producen y no de forma constante durante el plazo de ejecución.
5. El RD 1627/97 es un documento normativo de mínimos. Puede y debe exigirse en el contenido de los EBSS y ESS información complementaria que no figure de forma expresa en los Artículos 6 y 5 respectivamente del citado $\mathrm{RD}$, cuando sea precisa.

6. El incumplimiento normativo de contenidos mínimos, tanto en los EBSS con un 95,38\%, como en los ESS con un $91,80 \%$, es muy elevado, ya sea por omisión de contenidos, por su parcial desarrollo o un inadecuado tratamiento.

7. La justificación de incurrir en los supuestos b) o c) del Artículo 4.1 del RD 1627/97, debe hacerse mediante cálculos debidamente justificados. Para ello, deben utilizarse los datos del propio proyecto o en su caso, acudir a datos estadísticos de fuentes de solvencia que permitan una estimación con un mínimo de rigor profesional, teniendo siempre presente el convenio del sector y nunca asignando datos a bulto o de forma arbitraria.

8. Salvo excepciones, toda obra tiene riesgos evitables. Son medidas de primera intervención que una vez aplicadas, hacen que el riesgo desaparezca, no precisando ser incluidas en ningún otro análisis de riesgos.

9. Se confunde la valoración de la eficacia de las medidas preventivas, previstas para controlar los riesgos no eliminables, con la evaluación inicial de riesgos, que corresponde al desarrollo del PSS.

10. Siempre debe indicarse de forma expresa, la existencia o no de riesgos especiales de forma clara y diferenciada del resto de riesgos.

11. En los EBSS/ESS debe justificarse adecuadamente que las instalaciones existentes, en su caso, cumplen con las condiciones para ser utilizadas por el número máximo de trabajadores calculado. Debe constatarse que la utilización de dichas instalaciones, previa autorización, es compatible con la actividad de la dependencia.

12. Tanto en los EBSS como en los ESS, se deben identificar los centros asistenciales más cercanos y dejar claramente definidas las dotaciones sanitarias previstas en la obra.

13. Los ESS deben analizar la posibilidad o idoneidad de utilizar algunas de las medidas de seguridad que se instalan para el normal desarrollo de la obra y su posible utilización para trabajos posteriores de mantenimiento en el edificio.

14. El programa de trabajos de seguridad y salud es un documento que debe formar parte tanto el EBSS como el ESS, en función de las particularidades de la intervención, y en cumplimiento del Artículo 8 del RD 1627/97, como complemento al programa de trabajos del proyecto.

15. El EBSS debe incluir siempre, como mínimo, un plano general de ordenación de la obra, donde se refleje toda la información básica e imprescindible para definir la actividad preventiva.

16. Debe analizarse detalladamente el marco normativo que afecta a cada actuación, tanto por la particularidad de sus distintas actividades durante la obra como por la peculiaridad de la actividad que desarrolle el promotor en la dependencia donde se actúe. Se debe adaptar el marco legal a cada intervención de forma particularizada, evitar los listados generalistas y contemplar solo las normas que son de aplicación. 


\section{REFERENCIAS}

(1) Ministerio de la Presidencia (1997). Real Decreto 1627/1997 por el que se establecen disposiciones mínimas de seguridad y de salud en las obras de construcción. Boletín Oficial del Estado, $n^{0} 256$. España.

(2) Zapata Lobo, M. A. (2017). Modelo de gestión en materia de prevención de riesgos laborales en las obras de construcción de la Armada (Tesis Doctoral no publicada). E.T.S. Edificación. Universidad Politécnica de Madrid.

(3) Escrito de 16 de noviembre de 2015 de la Dirección de Infraestructura de la Armada y su anexo del Almirante Director de Infraestructura de la Armada.

(4) Comisión Nacional de la Competencia. Resolución Expediente S/0002/o7. Consejo Superior de Arquitectos de España de 29 de noviembre de 2010.

(5) Consejo General de la Arquitectura Técnica de España. Asesoría Jurídica. Informe sobre Titulaciones habilitantes para el desempeño de la coordinación de seguridad y salud y para la elaboración de los estudios de seguridad en las obras de edificación. Marzo de 2008.

(6) Jefatura del Estado (1995). Ley 31/1995 de Prevención de Riesgos Laborales. Boletín Oficial del Estado, nº 269. España.

(7) Jefatura de Estado (2017). Ley 9/2017 de Contratos del Sector Público, por la que se trasponen al ordenamiento jurídico español las Directivas del Parlamento Europeo y del Consejo 2014/23/UE y 2014/24/UE. Boletín Oficial del Estado, $\mathrm{n}^{0}$ 272. España.

(8) Martínez, Gonzalo. (2009, 27 de marzo). Sistema de Coordinación. Ponencia 17. Convención Nacional de la Arquitectura Técnica (CONTART). Albacete. Libro de actas.

(9) Ministerio de Empleo y Seguridad Social (2012). Guía técnica para la evaluación y prevención de los riesgos relativos a las obras de construcción del Instituto Nacional de Seguridad e Higiene en el Trabajo. http://www.insht.es/InshtWeb/ Contenidos/Normativa/GuiasTecnicas/Ficheros/g_obras.pdf. $2^{\text {a }}$ edición. Marzo 2012.

(10) Ministerio de Defensa (2011). Instrucción 55/2011 del Secretario de Estado de Defensa, por la que se aprueban las Normas sobre competencias y relaciones de los jefes de las bases, acuartelamientos o establecimientos con los órganos técnicos que en dichas dependencias realizan labores de dirección facultativa de obras. Boletín Oficial de Defensa, $\mathrm{n}^{0}$ 153. España. 